\title{
Violencia, migración y escritura en la diáspora: conversación con Ingrid Rojas Contreras
}

\author{
Astrid Lorena Ochoa Campo/ University of Wisconsin La Crosse
}

"We were refugees when we arrived to the U.S. You must be happy now that you're safe, people said. They told us to strive for assimilation. The quicker we transformed into one of the many the better. But how could we choose? The U.S. was the land that saved us; Colombia was the land that saw us emerge" (Rojas Contreras 2018, 2).

“Llegamos de refugiados a los Estados Unidos. 'Deben estar felices ahora que están a salvo', decía la gente. Nos dijeron que debíamos esforzarnos por adaptarnos. Mientras más rápido pudiéramos transformarnos en unos de tantos, mejor. Pero ¿cómo elegir? Estados Unidos era la tierra que nos había salvado; Colombia, la tierra que nos vio nacer" (Rojas Contreras 2019, 10).

\section{Colombianos en los Estados Unidos}

El número de colombianos que emigran ha ido aumentando considerablemente a través de los años. Desde el periodo de 1963-1973 hasta el año 2012, la cantidad pasó de 609.476 a 4.700.000 (Polo Alvis y Serrano López 2019, 318). Sobre la migración a Estados Unidos que comenzó en la década de 1950, se identifican tres olas: la primera, entre 1950 y finales de los 70, conformada por habitantes de las tres ciudades capitales más importantes del interior del país-Medellín, Cali y Bogotá - y asentada principalmente en Nueva York y su área metropolitana. Estas personas venían huyendo de $L a$ Violencia y eran de clase baja y media (Nasser De la Torre 2013, 296-97). La segunda ola comenzó en los últimos años de la década de 1970 y se extendió hasta mediados de la década de 1990. Este periodo coincide con el auge del narcotráfico. Los migrantes de esta ola provienen de todos los estratos socio-económicos (Nasser De la Torre 297). La tercera ola inició a mediados de los años 1990. Este grupo se distingue en cuanto está formado en su mayoría por profesionales jóvenes y otros individuos con mayor educación formal de clases media y alta (Cepeda 2003, 214). Recientes estudios sobre la diáspora colombiana alrededor del mundo se refieren a esta "tercera ola migratoria" como producto del "empeoramiento de las condiciones políticas, económicas y sociales en Colombia, a causa de flagelos como el narcotráfico, la violencia generalizada, el duro deterioro de la seguridad para los ciudadanos, entre otros" (Polo Alvis y Serrano López 2019, 316).

De acuerdo con el más reciente reporte del Pew Research Center, se estima que 1.2 millones de hispanos de origen colombiano vivían en los Estados Unidos en 2017. Estos incluían inmigrantes nacidos en Colombia y descendientes de colombianos. En estos momentos, los de origen colombiano constituyen la séptima población de origen hispano más grande viviendo en los Estados Unidos, lo cual equivale a un 2\% de la población total de hispanos en 2017. Desde el año 2000 la población de origen colombiano ha aumentado un $148 \%$. De estos, los nacidos en Colombia han aumentado un 99\%, de 383.000 en el año 2000 a 763.000 en 2017. Otros datos que arroja el informe indican que un $60 \%$ de los nacidos en Colombia se han convertido en ciudadanos norteamericanos; un $33 \%$ ha obtenido al menos un diploma de pregrado y su nivel de ingresos es un poco más alto que el promedio de la población hispana en los Estados Unidos; un 10\% de colombo-estadounidenses y un $12 \%$ de nacidos en Colombia vive en condiciones de pobreza y se concentran en los estados de Florida (31\%), Nueva York (14\%) y Nueva Jersey (12\%); y por último, en cuanto al idioma, un $62 \%$ de colombo-estadounidenses de 5 años de edad o más hablan solamente inglés en sus casas y un $57 \%$ de colombianos adultos se desenvuelven bien en el inglés (Noe-Bustamante, Flores y Shah 2019).

Aunque según estas estadísticas muchos colombianos poseen un estatus legal en los Estados Unidos, también existe un alto número de indocumentados. Ariana Ochoa Camacho (2016) subraya el discurso de doble criminalidad que genera la asociación de colombianos con el mercado global del narcotráfico y el cruce ilegal de fronteras (166). En su estudio de colombianos en la zona metropolitana de Nueva York, Ochoa Camacho examina el impacto de esta asociación negativa en el diario vivir de personas indocumentadas. La académica concluye que, para evitar el estigma, algunos colombianos recurren a estrategias como asimilarse o invertir asiduamente en la proyección de una imagen positiva del "buen" colombiano. Además, algunos se distancian de sus compatriotas y latinos en general, lo que resulta en "'aspirational whiteness,' which entails the embrace of Anglo-American cultural norms and ideas without the material benefits of propertied whiteness" (Ochoa Camacho, 172). A pesar del éxito de los pequeños negocios de colombianos en Jackson Heights que hace visible a la comunidad colombiana en Queens, Nueva York, esta visibilidad en la infraestructura no corresponde con la invisibilidad y falta de unidad en la comunidad colombiana: "Colombian migrants in New York experience the distress 
of migration and fight high levels of isolation and invisibility that characterize life in the United States as marked by solitude. In particular, undocumented Colombians live experiences of intense isolation" (Ochoa Camacho, 178). De un modo similar, Javier Castaño documenta las experiencias de colombianos en el barrio Corona de Queens. Allí, estos tienen publicaciones como Noticiero Colombiano y La Semana Metro y cuentan además con una estación de radio, La Nueva Radio Internacional, y dos canales de televisión por cable, Caracol y RCN (206). Como parte del esfuerzo por mostrar la cara linda de los colombianos, el líder cívico Rafael Castelar creó en 2000 el desfile en Northern Boulevard, el cual es actualmente el único evento público que representa a la comunidad colombiana en la ciudad de Nueva York, y que se lleva a cabo cada año en julio alrededor del día de la independencia de Colombia (Castaño 2017, 207). Castaño también observa cómo el estigma asociado con ser colombiano afecta la unidad de la comunidad para organizarse políticamente. El autor identifica el regionalismo y clasismo importados de Colombia como obstáculos para el progreso de actividades en pro de la unidad comunitaria (Castaño, 207).

Moviéndonos hacia el sur, María Elena Cepeda (2010) estudia la comunidad diaspórica de Miami en Musical ImagiNation: U.S. Colombian Identity and the Latin Music Boom. En su libro afirma que la música "provides a collective space for imagining Colombianidad outside traditional geopolitical borders" (Cepeda, 4). Así mismo, las obras de escritoras estadounidenses de origen colombiano contribuyen a imaginar una colombianidad en un contexto transnacional. En las dos últimas décadas, ha aumentado la producción literaria en inglés sobre experiencias de colombianos en diversas regiones de los Estados Unidos. Entre los relatos se destacan: Vida (2010) de Patricia Engel; Try to Remember: A Novel (2010) de Iris Gomez; A Cup of Water Under My Bed (2014) de Daisy Hernández; The Lucky Ones (2017) de Julianne Pachico (2017); Don't Come Back (2017) de Lina María Ferrerira Cabeza-Vanegas; Fruit of the Drunken Tree (2018) de Ingrid Rojas Contreras; Magical Realism for Non-Believers: A Memoir of Finding Family (2019) de Anika Fajardo; y Fiebre Tropical (2020) de Juliana Delgado Lopera. Por medio de sus historias de colombianos en los Estados Unidos, estas autoras capturan literariamente los aspectos cuantificados en las estadísticas anteriormente mencionadas. A continuación, se presenta una entrevista con Ingrid Rojas Contreras en la que se conversa sobre su emigración de Colombia, la experiencia de ser refugiada en los Estados Unidos, la situación migratoria en dicho país y su primera novela. Antes de la entrevista, doy algunos datos biográficos de la autora y traigo a colación algunos de sus artículos relacionados con la situación migratoria actual en los Estados Unidos.

\section{La escritora}

Ingrid Rojas Contreras es de origen colombiano, nacionalizada y radicada en los Estados Unidos. Ha escrito ensayos y cuentos que se han publicado en el New York Times, Buzzfeed, Nylon, Guernica, entre otros. Su primera novela, publicada en julio de 2018, se titula en inglés Fruit of the Drunken Tree y en español La fruta del borrachero (2019). El relato se nutre de las experiencias de su niñez en la ciudad de Bogotá durante las décadas violentas de los ochenta y noventa, y acontecimientos que forzaron la reubicación de su familia en Venezuela y posteriormente en los Estados Unidos en calidad de refugiados. Su novela recibió medalla de plata en First Fiction de los California Book Awards y el premio Editor's Choice del New York Times. En general ha tenido una buena acogida y aclamadas escritoras como Sandra Cisneros, Julia Álvarez y Patricia Engel han hecho notables comentarios de la novela. Además, Rojas Contreras ha recibido varios premios y becas de entidades reconocidas como Bread Loaf Writer's Conference, The Camargo Foundation y la National Association of Latino Arts and Culture. En la actualidad se desempeña como escritora visitante en Saint Mary's College. 1

Dada su condición de inmigrante en los Estados Unidos, Rojas Contreras se basa en esta experiencia en sus escritos y, además, a través de algunos de ellos, se ha manifestado públicamente en contra de los abusos de la administración del presidente Donald Trump. Con su primera novela, Fruit of the Drunken Tree, incursiona en la exploración de las vivencias de colombianos en los Estados Unidos, a la cual han contribuido especialmente autoras como Patricia Engel y Daisy Hernández. Teniendo en cuenta la definición de "Latinx literature" como "a transnational, pluralistic, heterogeneous, and dynamic category that broadly considers the writings of diverse peoples with cultural ties to Latin America residing in the United States" (Smith y Franco 2018, 9), la novela de Rojas Contreras se puede considerar representante de esta categoría. Además, con la traducción al español de esta obra la autora no solo intenta alcanzar a un público hispanohablante en Colombia, sino también en los Estados Unidos y el resto del mundo.

En su ensayo autobiográfico "Nomadic", el cual hace parte de la colección Wise Latinas: Writers on Higher Education (2014), Rojas Contreras reflexiona sobre su experiencia al iniciar sus estudios universitarios en Chicago y enfrentarse al crudo invierno de la ciudad por primera vez. Allí describe que su familia era una "typical South American family, was mainly Catholic, but we also believed in the divination of dreams, the reading of the tarot spread, the reading if tealeaves, protections and amulets, the evil eye" (38). También deja clara la razón de su migración a los Estados Unidos: "Studying in Colombia was out of the question. I had promised my father I would study oversees after the guerrillas kidnapped two of my uncles" (39). El secuestro, material fructífero para 
la ficción, aparece entonces en el capítulo de su novela titulado "Ghost House", previamente publicado como cuento en la revista Guernica en 2014. Para esta escritora, la violencia no solo hace parte de sus recuerdos de infancia en Colombia, sino que también se manifiesta como una constante en la infancia de sus padres. En un artículo de opinión sobre el significado que tiene para ella la palabra "home", explica cómo aun en Bogotá se sentía entre dos mundos, sentimiento que luego permea su conciencia de inmigrante en Estados Unidos:

I never felt like I belonged in my childhood home in Bogotá. Ours was a three-story house in a gated community built to keep things out: crime, the tin-and-cardboard shelters of the Displaced, the kidnappings, and the car bombs [...] My family had no money. My father's family had been displaced by La Violencia, a decade-long civil war between conservatives and liberals, during which their small plot of land was torched and their animals slaughtered $[\ldots]$ My mother grew up stealing cows and cabbages at her own mother's behest so that her eight brothers and sisters could survive. In many ways, my parents were what our gated community wanted to keep out, and I was born at the border of these two things. (Rojas Contreras 2018, "For Debut")

En su artículo publicado en el HuffPost, luego de convertirse en ciudadana norteamericana, justo el día en que Trump hizo su comentario denigrante sobre algunos países como "shitholes", Rojas Contreras nuevamente subraya cómo se vive en los intersticios como inmigrante: "being an immigrant is a malleable place, an in-between, a pending state. Through our oath, we were leaving that place. We were becoming something politically tangible" (Rojas Contreras 2018, "I Became"). Ya en una pieza anterior, escrita para USA Today, Rojas Contreras se había manifestado sobre la continua zozobra que experimentan no solo los inmigrantes indocumentados y los que están en busca de asilo, sino también aquellos a quienes ya se les ha autorizado su permanencia en el país como ciudadanos: "More than others, this particular administration is intent on not letting naturalized citizens forget that we are foreign-born, and it is intent on reminding us with how much derision it beholds this fact. Our foreign birth is a perpetually open door through which we can always be kicked out" (Rojas Contreras 2018, "Donald"). Así, esta escritora se convierte en una vocera más en contra de las miríadas de injusticias contra migrantes cometidas por la administración actual en los Estados Unidos.

\section{La entrevista}

La novela Fruit of the Drunken Tree narra la historia de una familia de clase media de Bogotá que, luego del secuestro del papá por parte de la guerrilla, debe migrar primero a
Venezuela y luego a Estados Unidos, donde son bien recibidos en una comunidad de refugiados. Las dos narradoras principales son la hija menor de la familia, Chula, y Petrona, la niña empleada de servicio. A través de estas dos voces se presentan versiones diferentes de algunos sucesos enmarcados en el panorama violento de Colombia durante las décadas de los ochenta y noventa. A propósito de la publicación de su primera novela, me reuní con Ingrid Rojas Contreras para conversar sobre diversos temas de la obra, su experiencia como inmigrante y la escritura en la diáspora. Nuestra charla tuvo lugar el 7 de septiembre de 2018 en la cafetería Liberty Arts Coffee en Winston, Salem, Carolina del Norte.

\section{ALOC: ¿Te definirías como una escritora latina o como una escritora colombiana? ¿Qué audiencia tienes en mente cuando escribes?}

IRC: El ser emigrante ha sido el centro de la manera en que empecé a escribir y el centro de la manera en que continúo escribiendo. Entonces estoy entre países y estoy entre culturas y ese lugar de no estar ni aquí ni allá es la manera en que me definiría.

\section{ALOC: Cuéntanos un poco cómo te convertiste en escritora, ¿qué te llevó a esta carrera?}

IRC: Desde que era chiquita, cada vez que llegaba el periódico a la casa, yo corría y recogía el periódico y olía la tinta. El olor me encantaba, el olor de la tinta. Me encantaban los libros y siempre fue algo que me atrajo, pero no empecé a escribir sino hasta cuando tenía como catorce años y mi familia y yo nos fuimos a Venezuela. Fue en ese entonces que nos fuimos a un pueblito que se llama Maturín, y pues de Bogotá a Maturín había muchas diferencias y el verme sola y el verme en un lugar desconocido, me llevó a escribir en un cuaderno. Empecé a escribir en inglés desde ese entonces. La escritura para mí siempre estuvo conectada con ese momento de migración. Empecé a escribir en inglés, que no es mi lengua materna, pero era un lenguaje que mis papás no podían leer, era un lenguaje secreto que era solo para mí, nadie me iba a entender. Con mi cuaderno me sentaba en diferentes partes de mi casa y empezaba a describir cosas que estuvieran alrededor. Me sentaba por ejemplo al lado de un vaso de agua y describía todo lo que veía... la luz, el vaso, la mesa. Lo otro que escribía eran cuentos como de un párrafo, súper cortos.

\section{ALOC: Micro-cuentos}

IRC: Micro-cuentos, sí, y la actividad de escribir era algo que me ayudaba a entender mi alrededor y también me ayudaba a entender la emoción de dejar tu país y de ir a un país nuevo, la emoción de hacer vida nueva, la emoción de no estar con tus raíces. Entonces desde ese momento la escritura 
para mí... me calmaba una sed que nunca se me calmó de ninguna otra manera.

ALOC: Imagino que al mismo tiempo que estabas escribiendo, como es el caso de muchas personas que se dedican a la escritura, también estabas devorando libros, ¿no?. Entonces, ¿qué tipo de libros te inspiraron desde esa época?

IRC: Sí, en ese entonces yo estaba leyendo todos los libros de Gabriel García Márquez. Me los leí todos. Isabel Allende me gustaba mucho en ese entonces. Don Quijote es el primer libro que me acuerdo haber leído. Lo leí en Colombia cuando tenía doce años. Ese año tuvimos un profesor de literatura en español que pensó que podíamos leerlo. Yo creo que nadie en la clase pasó más del primer capítulo, pero para mí fue un libro en que encontré humor, y me asombré tanto que un humor de hace tantos años todavía me pareciera a mí chistoso-una niña en Bogotá en los $90 \ldots$ me pareció mágico. Desde ese momento me volví lectora empedernida.

ALOC: ¿Qué libros te han impactado más en los últimos cinco años, por ejemplo?

IRC: En los últimos cinco años, un libro que me impactó muchísimo y en el que pienso mucho es un libro de poesía que se llama Look (2016), de la poeta Solmaz Sharif. Ella es de Irán, y es un libro que usa el diccionario del Departamento de Defensa de los Estados Unidos, y usa las definiciones que ellos tienen para diferentes palabras en poemas acerca de su vida personal, pero también de su tío, que estuvo prisionero en Irán. Entonces el título, la palabra "look", para el diccionario de Defensa, no es mirar, no es ver, sino que significa el momento en que alguien pisa una mina quiebrapatas pero antes de que retire el pie. Ese momento se llama "look". Entonces fue un libro que me impactó mucho por la manera en que Sharif analiza una palabra y le da tantos significados.

ALOC: Hablemos entonces de tu novela, ifelicitaciones por la publicación de Fruit of the Drunken Tree y su edición en español, La fruta del borrachero! ¿Cómo llegas a este momento o a la decisión de escribir esta novela?

IRC: Estaba en Chicago y en realidad no fue una decisión que tomé, sino que la historia me acechó y no me dejaba en paz. Yo trataba de escribir algo más y siempre lo que escribía me llevaba a la historia de la novela. Por muchos años traté de no escribirla y traté de escribir otras cosas. Cuando por fin me di por vencida y empecé a escribir la novela, fue muy sanador.

ALOC: La novela inicia cuando Chula está mirando la fotografía que Petrona le manda:
On the back there's a date stamp of when the photo was printed-and because when I count back nine months it falls exactly on the month my family and I fled from Colombia and arrived in L.A., I turn back the photograph to look intently at the baby, to register every wrinkle and bulge around the dark hole of his gaping mouth, to decide whether he is crying or laughing, because I know exactly where and how he was conceived and that's how I lose track of time, thinking it was my fault that the girl Petrona was just fifteen when her belly was filled with bones... (Rojas Contreras 2018, 2)

¿Cómo decidiste este comienzo y por qué rememorar a través de una fotografía?

IRC: Esa parte de la novela surgió al final cuando estaba escribiendo. Quería tener un momento que fuera una ventana que abriera el camino, el paso a esta historia. Leyendo el manuscrito que tenía, pensé que lo más importante para Chula era recordar y lo más importante para Petrona era el querer olvidar. Y esos dos deseos se conectaban en una comunicación, en una carta. Pensé que este era el motor de cada personaje, y entonces empezar con una carta en la que estos dos motores se encuentren era la manera perfecta de comenzar.

ALOC: Esta novela se publica casi dos años después de haberse firmado los acuerdos de paz con las FARC en Colombia y tú mencionas al final de tu novela, en la nota del autor, que en el momento de la escritura ya muchos de estos grupos se han desmovilizado. ¿Cómo crees que tu novela está siendo recibida en este momento en el que no se quiere hablar más de guerras sino de paz y vuelves en tu novela a varios momentos críticos de esta historia del conflicto armado en Colombia?

IRC: En Colombia, es mi experiencia que nunca queremos mirar hacia atrás y siempre queremos mirar hacia delante, pero el no mirar hacia atrás, el no entender, o digerir qué fue lo que nos pasó antes, no nos ayuda mucho. Para mí es más importante entender todas las facetas de algo que pasó, a simplemente mirar a un futuro que no ha pasado y que no sabemos cómo va a ser. Pero también la novela está basada en una historia personal que me pasó, y mi deseo de escribir la novela nació de un lugar muy personal y muy emocional para mí. Yo quería entender este momento que hizo que mi familia y yo nos fuéramos de Colombia.

ALOC: Esto que estás diciendo me hizo pensar en Chula. Muy temprano en la novela, ella está hablando de su papá a quien admira tanto, que quiere saber tanto como él de todo o mucho sobre el conflicto armado, cuáles son los grupos 
paramilitares, cuáles son los grupos guerrilleros, y está confundida:

Papá was like a walking encyclopedia. He boasted that he could name at least a third of the 128 paramilitary groups in Colombia [...] He also said he knew some of the names of the groups within the death squadrons, the narco-paramilitary [...] the regular guerrillas [...] but his specialty was the paramilitary. I tried hard to be like Papá, but no matter how much of an effort I made, I couldn't even grasp the simplest of concepts-what was the difference between the guerrilla and the paramilitary? What was a communist? Who was each group fighting? (Rojas Contreras 2018, 17)

Desde una mirada infantil, Chula no puede establecer la diferencia entre los motivos de los grupos para combatir. ¿Tú crees que esta confusión está presente en la mente de muchos colombianos? ¿Por qué expresar esto a través de la voz de la hija?

IRC: En Colombia hay muchas noticias que no se saben sino hasta años después $y$, cuando yo estaba escribiendo la novela, quise que se sintiera como la experiencia real de cómo fue vivir en los noventas. Cuando estaba escribiendo la novela, Google estaba escaneando todos los periódicos del mundo y los estaba poniendo en línea, entonces en ese año, cuando ya tenía todo el bosquejo de la novela, me senté y leí todos los periódicos de todos los días, desde el 89 hasta el 95-pero también leí los periódicos de años después y había muchas cosas que no se supieron en el momento. En Colombia hay tantos secretos políticos, que nunca se entiende en el momento qué es lo que está pasando. Pensar en la novela y en una niña que está viviendo en este tiempo y qué es lo que puede entender un niño en ese tiempo, fue un ejercicio creativo muy interesante para mí. Lo más divertido de escribir fueron esos momentos en que Chula estaba escuchando un pedacito de una noticia y la manera en que lo malinterpretaba, o la manera en que estaba confundida acerca de qué es un comunista y qué es un paramilitar y qué es un guerri1lero. Cuando fui a Colombia hace años, algo que me dio a entender cómo un niño puede estar confundido con su alrededor, fue que estaba visitando a una tía y mis primos, que eran pequeños en ese entonces, vieron pasar a un militar en camuflaje. Ellos inmediatamente se escondieron, con miedo, y yo les estaba tratando de explicar que ellos eran soldados de las Fuerzas Armadas, no tenían por qué tener miedo. Pero me di cuenta de que el lenguaje visual es casi todo lo que un niño puede entender - si ven a alguien en camuflaje, solo saben que puede ser alguien peligroso.
ALOC: ¿Cómo fue esta experiencia de investigar para la novela? ¿Cómo te sentías al volver tu mirada a estos hechos de la realidad de Colombia?

IRC: Cuando leí los periódicos, los leí como si estuviera leyendo una novela. En una cartulina hice una gráfica de lo que me imaginaba era la trayectoria desde un principio hasta un fin, empezando con el asesinato de Luis Carlos Galán y terminando con Pablo Escobar cuando lo mataron en ese techo en Medellín. Para mí esa historia tiene movimiento y tiene su clímax propio y también están estos dos personajes que son como las dos caras de Colombia. Luis Carlos Galán fue alguien en quien muchos colombianos tenían fe, le decían "el futuro de Colombia". Era algo que decían mucho en los periódicos. Lo que me pareció más impactante leyendo los periódicos como si fueran una novela es que, en los dos funerales de estas dos personas, la gente cantó lo mismo. La gente cantó "se vive, se siente, Galán está presente" en el funeral de Galán, y en el funeral de Pablo Escobar se cantó "se vive, se siente, Escobar está presente". Siempre me gustó mucho la política, la manera en la que se refleja en las vidas de las personas que se ven afectadas, y para mí esa es la pieza más importante. Cómo afecta todo este entorno, cómo afecta a las personas, pero lo que más me interesa es cómo afecta a las mujeres y a las niñas jóvenes. En ese entonces, estaba entre visas de estudiante viviendo en Chicago y no podía viajar a Colombia, y emocionalmente para mí leer los periódicos y empezar a escribir esta novela fue una manera de volver sin poder ir físicamente. Entonces fue como una manera muy larga de recordar a Colombia.

ALOC: Pensando en la niña víctima de un carro bomba en el capítulo 5, “The Dead Girl's Shoe”, los juegos de guerra de las niñas con sus Barbies, y otros acontecimientos que están en la imaginación de las personas en Colombia y en el exterior a través de libros, películas y series de televisión, ¿cómo fue tu experiencia al contar algunas historias alrededor de la figura de Pablo Escobar?

IRC: Lo que yo he observado es que casi todas las novelas o las películas acerca de Pablo Escobar siempre lo ponen como un personaje principal y siempre se empeñan en entender qué estaba pensando él, qué hacía día a día, cómo era su vida amorosa, y para mí al vivir en Colombia, Pablo Escobar no era un personaje principal. Pablo Escobar era el clima de Colombia, algo que estaba en el fondo. Al escribir esta novela quería que eso fuera el fondo, quería que la historia no estuviera centrada en los agresores de la política colombiana, pero sí en las víctimas de la violencia y las víctimas jóvenes de la violencia.

ALOC: Y, hablando de las víctimas, estas están distribuidas en todas las clases. Tenemos a Petrona, que es una joven de clase baja, luego está la familia de Chula, que es de clase 
media alta; están estos personajes como la Oligarca y la Soltera, que están en un estrato más alto pero que también tienen historias, secretos, pero todas están de alguna manera afectadas por la violencia. ¿Cómo surgió esto al escribir tu novela?

IRC: Vino de algo que entendí cuando era muy chiquita. Mis dos padres vienen de familias de clase baja del nororiente de Colombia, pero nosotros vivimos en una casa en un conjunto cerrado en Bogotá. La experiencia de mi niñez fue de viajar entre mi casa de clase media a ir a visitar a mi familia en sus barrios. Entonces pensé que era una experiencia singular, el de viajar entre un mundo y el otro. Para mí fue muy importante poder imaginar eso en la ficción.

ALOC: Anteriormente hablabas de estar "entre papeles", y me interesa el momento en que Chula y su familia llegan a Estados Unidos y empiezan a establecer relaciones con la comunidad de migrantes porque es un episodio impactante de la novela y muy relevante en la actualidad. ¿Cómo fue la experiencia de escribir sobre refugiados en tu novela?

IRC: Lo que más me llamó la atención cuando llegué por primera vez a Estados Unidos fue que hay el mundo de los norteamericanos y hay un mundo de los inmigrantes. Cuando los inmigrantes se encuentran y se conocen y empiezan a hablar entran en un mundo totalmente distinto. Noté que recordar es algo que se desea mucho, recordar el país perdido es algo que queremos hacer con muchas ansias, y al mismo tiempo hay otro deseo que compite, que es el de hacer una nueva vida y dejar todo atrás para convertirse en alguien más. El inmigrante se encuentra en una oportunidad única: nadie te conoce y por primera vez puedes cambiar tu estilo, puedes volverte otra persona.

\section{ALOC: como reinventarte.}

IRC: Exacto, sí. Desde que pasó 9/11, el gobierno de Estados Unidos ha limitado quién puede venir a Estados Unidos y quién puede ser refugiado. Cuando Chula llega a Estados Unidos no ha pasado el 9/11, pero si ella hubiera llegado un poco después, hubiera habido otras reglas. Por ejemplo, si tú le hubieras dado de comer a un paramilitar o a un guerrillero que te dijo denos de comer, denos su cosecha o lo matamos, el gobierno de Estados Unidos lo hubiera mirado como un aporte de sustancia material a un grupo terrorista y no te hubiera permitido solicitar entrada como refugiado. El tipo de persona que tiene derecho a pedir asilo se ha ido reduciendo ... hasta el punto de que ahora, en la imaginación popular, cruzar una frontera y pedir asilo está en contra de la ley-aunque no lo está.

ALOC: En tu novela describes la casa de Chula como un "reino de mujeres"; tu novela tiene personajes femeni- nos como Chula, Petrona, Alma, la madre, Cassandra, la hermana, y en algún momento Chula dice que su mamá les ha dicho que ellas son feministas:

\begin{abstract}
Always when he came home Papá upset our kingdom of women [...] Papá had strange rules about hair and how long it should be. Mamá said it was all part of a sordid belief system called machismo. Mamá said Papá was a machista. On the other hand, Mamá, Cassandra, and I were feministas. Meaning, if I wanted to have short hair, Mamá would let me and Cassandra would like it. (Rojas Contreras 2018, 49)
\end{abstract}

Ya que dices que tu novela está basada en tu historia personal, me preguntaba si tu mamá usó la palabra "feminista", o fue algo que quisiste resaltar y por qué.

IRC: Yo crecí al lado de mis tías y al lado de mi mamá y cada vez que ellas se sentaban a contar historias, yo iba pegada para saber qué era lo que decían. Mi mamá en la vida real es una mujer muy fuerte, muy emprendedora, pero nadie le enseñó a ser así. Cuando ella era chiquita, una de las historias de ella que más me impactó-que escuché cuando estaba creciendo y me estaba volviendo mujer y estaba pensando en cómo quería ser mujer - una de las historias que ella me contó fue que, en su familia, su mamá le decía que ella tenía que lavar y planchar la ropa de sus hermanos. Sus hermanos podían tener 13, 14 años, y ella tenía 9, y ella tenía que lavar la ropa y lavar los platos, y ella dijo que no. Le pegaron con correa y todo, y ella seguía diciendo que no lo iba a hacer. Viendo que su mamá no la escuchaba, se cortó su propio cabello y le dijo a su mamá, "Ahora soy niño, no voy a lavar y no me toca hacer nada". Cuando ella me contó esa historia, entendí que había reglas que se podían romper. Entonces para mí ese fue un momento que me definió mucho como persona, y en mi novela quería honrar a las mujeres con las que crecí y la manera en que luchaban en un país que, en ese entonces, y todavía, continúa siendo muy machista, y que no está interesado en que la mujer tenga un rol intelectual o de líder.

ALOC: Continuando con esto de las relaciones, ¿describirías la relación entre Chula y Petrona como una relación de amistad?

IRC: Es una amistad complicada ¿cierto? Sí hay amistad, pero lo que quería plasmar en el libro es que cuando es una amistad entre clases, este hecho siempre va a estar en el medio. El hecho que Petrona sea empleada en la casa de Chula siempre va a envenenar la relación. En el libro quería explorar la manera en que las clases y la circunstancia política afectan a cada personaje, y cómo afectan las relaciones entre los personajes. Ellas se quieren mucho, son amigas, pero hay momentos en los que no se entienden porque están viviendo 
en mundos muy distintos. Es algo que empieza a desboronar la relación.

\section{ALOC: Finalmente, ¿en qué estás trabajando en estos} momentos?
IRC: Estoy trabajando en una memoir. Es acerca de mi abuelo, que era curandero en Colombia y acerca de lo que se hereda entre generaciones.

\section{Obras citadas}

Castaño, Javier. 2017. "Ecuadoreans and Colombians in New York". En Latinos In New York: Communities in Transition. Second edition, editado por Sherrie L. Baver et al., 192-219. Notre Dame: University of Notre Dame Press.

Cepeda, María Elena. 2010. Musical ImagiNation: U. S-Colombian Identity and the Latin Music Boom. Nueva York: New York University Press. Ebook Central.

---. 2003. "Shakira as the Idealized Transnational Citizen: A Case Study of Colombianidad in Transition". Latino Studies 1 (2): 211-32.

Delgado Lopera, Juliana. 2020. Fiebre Tropical. Nueva York: The Feminist Press.

Engel, Patricia. 2010. Vida. Nueva York: Black Cat.

Fajardo, Anika. 2019. Magical Realism for Non-Believers: A Memoir of Finding Family. Minneapolis y Londres: University of Minnesota Press.

Ferrerira Cabeza-Vanegas, Lina María. 2017. Don`t Come Back. Columbus: Mad River Books, The Ohio State University.

Gomez, Iris. 2010. Try to Remember. Nueva York y Boston: Grand Central Publishing.

Hernández, Daisy. 2014. A Cup of Water under My Bed: A Memoir. Boston: Beacon Press.

Nasser De la Torre, Michelle Rocío. 2013. "Bellas Por Naturaleza: Mapping National Identity on US Colombian Beauty Queens". Latino Studies 11 (3): 293-312.

Noe-Bustamante, Luis, Antonio Flores y Sono Shah. 2019. "Facts on Hispanics of Colombian Origin in the United States, 2017”. Pew Research Center, septiembre 16, 2019. https://www.pewresearch.org/hispanic/fact-sheet/u-s-hispanics-factson-colombian-origin-latinos/

Ochoa Camacho, Ariana. 2016. "Living with Drug Lords and Mules in New York: Contrasting Colombian Criminality and Transnational Belonging". En The Immigrant Other: Lived Experiences in a Transnational World, editado por Rich Furman, Greg Lamphear y Douglas Epps, 166-179. Nueva York: Columbia University Press.

Pachico, Julianne. 2017. The Lucky Ones. Nueva York: Spiegel and Grau.

Polo Alvis, Sebastián, y Enrique Serrano López. 2019. "La diáspora silenciosa: Estudios sobre la tercera ola de migraciones colombianas al exterior (2005-2015)". Desafios 31 (1): 311-46.

Rojas Contreras, Ingrid. 2018. "Donald Trump's Denaturalization Task Force Is a New Tay to Threaten the American Dream." USA Today, julio 24, 2018. https://www.usatoday.com/story/opinion/2018/07/24/donald-trump-denaturalization-goalsthreaten-american-dream-column/815592002/.

---. 2018. "For Debut Novelist Ingrid Rojas Contreras, Home is What You Carry with You." Architectural Digest, julio 30, 2018, https://www.architecturaldigest.com/story/ingrid-rojas-contreras-fruit-of-the-drunken-tree.

---. 2018. Fruit of the Drunken Tree: A Novel. Nueva York: Doubleday. 
---. 2014. "Ghost House.” Guernica, octubre 15, 2014. https://www.guernicamag.com/ghost-house/.

---. 2018. “I Became an American The Day Trump Made His 'Shithole Countries' Comment." HuffPost, enero 16, 2018 (Updated 18 January, 2018). https://www.huffpost.com/entry/new-americans-shithole-comment_n_5a5d02ede4b0fcbc3a1 $\underline{2 a b 3 b}$.

---. 2019. La fruta del borrachero. Traducido por Guillermo Sánchez Arreola. Salamanca: Impedimenta.

---. 2014. "Nomadic.” En Wise Latinas: Writers on Higher Education, editado por Jennifer De Leon, 38-53. Lincoln y Londres: University of Nebraska Press.

Smith, Amanda M. y Alfredo Franco. 2018. "Introduction: The Politics of Latinx Literature Today". Chiricú Journal: Latina/o Literatures, Arts, and Cultures 2 (2): 5-19.

\section{Nota}

1. Para más información sobre Ingrid, visite su sitio web https://www.ingridrojascontreras.com/ 